\title{
INAPPROPRIATE PRESCRIBING OF BENZODIAZEPINES BY DOCTORS IN NSW
}

\section{Pia Salmelainen}

Policy Analyst

Pharmaceutical Services Branch

Benzodiazepines are widely used, most commonly to treat short-term conditions such as anxiety and insomnia. Although they are generally effective in treating these conditions, and are relatively safe to use, physical dependence and symptoms of withdrawal can occur, even when taken in recommended therapeutic doses. Benzodiazepines are also liable to be abused by some people, such as alcohol- and heroin-dependent persons.

For these reasons benzodiazepines should be prescribed with some caution. To assist doctors, the Pharmaceutical Services Branch has developed guidelines called Recognising and Handling Patients Liable to Abuse Benzodiazepines. The guidelines include information about how to recognise and deal with patients who seek benzodiazepines and who are likely to abuse benzodiazepines obtained on prescription. It also outlines a doctor's obligations under the Poisons and Therapeutic Goods Regulation. These include prescribing within therapeutic standards, and maintaining appropriate records. It should be noted that patients are legally obliged to inform a doctor of the quantity of any benzodiazepines prescribed to them within the previous two months.

Each year a number of doctors come to the attention of the Pharmaceutical Services Branch of the NSW Department of Health because they prescribe benzodiazepines inappropriately. This includes: prescribing at rates in excess of the recommended therapeutic dose, prescribing for inappropriate purposes, prescribing to persons liable to abuse benzodiazepines, and prescribing benzodiazepines long-term. This paper presents findings from an examination of the benzodiazepine-prescribing practices of doctors who were investigated by the Pharmaceutical Services Branch. It highlights the problems in this area of prescribing, and describes guidelines that have been developed to assist doctors.

\section{METHOD}

Data were extracted from investigation files held by the Branch. The sample, selected for convenience, comprised 21 doctors who had come to the attention of the Branch during the period 1995 to 1997 , because of inappropriate benzodiazepine prescribing. It is estimated that the sample represented between five and 10 per cent of all doctors investigated some time during 1995-97 for their prescribing of benzodiazepines.

The majority of the sample (18) practised in the Sydney metropolitan area. Most of the doctors (20) were general practitioners. The doctors in the study were investigated about their prescribing to a variable number of patients, ranging from 1 to 113 (median=2).

\section{FINDINGS}

All of the doctors in the sample engaged in more than one inappropriate prescribing practice.

\section{Prescribing at rates in excess of the recommended therapeutic dose}

At least 10 doctors prescribed benzodiazepines at rates indicating daily doses in excess of the recommended therapeutic dose. Generally in these cases, the amount prescribed was three or more times the recommended therapeutic dose. In one case a patient had been prescribed what appeared to be 50 times the recommended therapeutic dose.

\section{Prescribing for inappropriate purposes}

At a follow-up interview 13 doctors indicated they had prescribed benzodiazepines to a patient because the patient had requested the particular drug. Often in these instances the patient requested the drug to deal with a condition they claimed to have, such as a sleeping problem or pain. Sometimes the patient claimed that only one particular benzodiazepine was effective.

Six doctors said they prescribed benzodiazepines because they were trying to control the patient's benzodiazepine dependency problem. However, the doctors were not able to control their patients' access to benzodiazepines, as some patients sought further prescriptions from several different doctors (that is, were 'doctor shopping').

\section{Prescribing to persons liable to abuse benzodiazepines}

At least 16 doctors prescribed benzodiazepines to persons who were on the methadone maintenance program, or to persons whom the doctor knew were-or suspected ofabusing drugs. Most of the doctors treating patients who were on the methadone maintenance program did not take appropriate action, such as asking the patient about their methadone treatment, or contacting the patient's methadone prescriber, or seeking assistance from the Pharmaceutical Services Branch.

In those instances where the doctor knew a patient had a history of drug abuse, it was uncommon for the doctor to refer the patient to a drug and alcohol specialist.

Some doctors suspected their patients were seeing other doctors for benzodiazepines, while others appeared to be unaware that their patients were 'doctor shopping'. Several doctors in this latter group expressed surprise or shock at 
hearing from the investigator that their patient was a 'doctor shopper'. Only occasionally did a doctor indicate to the investigator that they asked their patients whether they were seeing other doctors.

\section{Prescribing benzodiazepines long-term}

All doctors in the study had prescribed benzodiazepines on a long-term basis. Over half of the doctors had prescribed benzodiazepines to one or more patients for a period of at least 12 months. One patient had been prescribed benzodiazepines for a 10 -year period.

\section{DISCUSSION}

While benzodiazepines are generally effective and safe drugs to use, there is a significant risk of a patient becoming dependent or experiencing withdrawal after a relatively short period of use. Furthermore, there is a small but significant group of patients in the community who abuse benzodiazepines.

The Royal Australian College of General Practitioners has produced a publication, called Anxiety and Insomnia: Think Twice Before Prescribing, which outlines principles of good management for the prescribing of benzodiazepines. ${ }^{1}$ It includes information on the management of anxiety and insomnia, withdrawal from benzodiazepines, and the prevention of dependence. There is also a section on drug misuse that discusses prescribing in relation to habitual drug users.

\section{REFERENCE}

1. Mant A, de Burgh S, Letton T, Shaw J. Anxiety and insomnia: think twice before prescribing. Melbourne: The Royal Australian College of General Practitioners, 1997. it

To obtain a copy of Recognising and Handling Patients Liable to Abuse Benzodiazepines (publication number TG199) write to the Duty Pharmacist, Pharmaceutical Services Branch, NSW Department of Health, PO Box 103, Gladesville, NSW 1675; or telephone (02) 9879 3214. The Duty Pharmacist is also able to supply doctors with written information to assist them with patients seeking other drugs of dependence.

\section{HIV INFECTION, AIDS, HEPATITIS C, AND SEXUALLY TRANSMISSIBLE INFECTIONS IN AUSTRALIA: NATIONAL SURVEILLANCE RESULTS TO DECEMBER 1998}

\section{Ann McDonald \\ National Centre in HIV Epidemiology and Clinical Research, Darlinghurst}

The third annual surveillance report HIV-AIDS, Hepatitis $C$ and Sexually Transmissible Infections in Australia Annual Surveillance Report 1999 was released in October 1999. ${ }^{1}$ The report, framed in the context of the third National HIV-AIDS Strategy 1996-97 to 1998-99, provides a comprehensive summary of current knowledge on the pattern of HIV infection, AIDS, hepatitis C, and sexually transmissible infections in Australia. It includes information on:

- time trends in diagnoses of those infections;

- incidence and prevalence in populations at higher or lower risk of HIV infection, such as gay and other homosexually-active men, indigenous people, people who have injected drugs, and people at risk through heterosexual contact only;

- patterns of treatment for HIV infection;

- patterns of risk behaviour for HIV and related infections.

The main findings are presented as text, supported by figures. The data are presented as tables and follow the main report. This article outlines key results from the report.

\section{TRENDS IN DIAGNOSES OF HIV INFECTION AND AIDS}

The annual number of diagnoses of AIDS in Australia has dropped from a peak of 950 cases in 1994 to an estimated 348 in 1998 (Figure 3). The annual number of diagnoses of HIV infection has also continued to decline, to around 660 in 1998. However, the number of documented cases of newly acquired HIV infection in 1993-1998 has remained stable at 150-200 infections per year, providing a lower limit to the number of cases of HIV transmission that have actually occurred in Australia each year. Allowing for undocumented cases, it is estimated that 450 cases of newly acquired HIV infection occur in Australia annually.

The number of people living with HIV infection in Australia was estimated to be 11,800 by the end of 1998 including 2,430 people living with AIDS. The number of people living with HIV infection in Australia is estimated to gradually increase to around 13,000 by the year 2003 .

Transmission of HIV in Australia continues to occur primarily through sexual contact between men. No evidence was available of recent change in rates of HIV transmission through male homosexual contact, nor any increase in the very low rates of transmission through 